\title{
Stochastic logistic fuzzy maps for the construction of integrated multirates scenarios in the financing of infrastructure projects
}

\author{
Juan David Gonzalez-Ruiz ${ }^{\mathrm{a}, *}$, Alejandro Peña ${ }^{\mathrm{b}}$, Eduardo Alexander Duque ${ }^{\mathrm{c}}$, Alejandro Patiño ${ }^{\mathrm{b}}$, \\ Francisco Chiclana ${ }^{\mathrm{d}, \mathrm{e}}$, Mario Góngora ${ }^{\mathrm{d}}$ \\ ${ }^{a}$ Finance and Sustainability Reserch Group, Departamento de Economía, Universidad Nacional de Colombia, Medellín, \\ Colombia \\ ${ }^{b}$ Computational Intelligence and Automation Research Group, EIA University, Envigado, Antioquia, Colombia \\ ${ }^{c}$ Electronics Sciences 8 Informatics Research Group, Institución Universitaria Pascual Bravo, Medellín, Colombia \\ ${ }^{d}$ Institute of Artificial Intelligence (IAI), DeMontfort University, Leicester, UK \\ ${ }^{e}$ Department of Computer Science $\mathcal{G}$ Artificial Intelligence, University of Granada, Spain
}

\begin{abstract}
In general, the development of economic infrastructure systems requires a behavioural comprehensive analysis of different financial variables or rates to establish its long-term success with regards to the Equity Internal Rate of Return (EIRR) expectation. For this reason, several financial organizations have developed economic scenarios supported by computational techniques and models to identify the evolution of these financial rates. However, these models and techniques have shown a series of limitations with regard to the financial management process and its impact on EIRR over time. To address these limitations in an inclusive way, researchers have developed different approaches and methodologies focused on the development of financial models using stochastic simulation methods and computational intelligence techniques. This paper proposes a Stochastic Fuzzy Logistic Model (S-FLM) inspired by a Fuzzy Cognitive Map (FCM) structure to model financial scenarios. Where the input consists in financial rates that are characterized as linguistic rates through a series of adaptive logistic functions. The stochastic process that explains the behaviour of the financial rates over time and their partial effects on EIRR is based on a Monte Carlo sampling process carried out on the fuzzy sets that characterize each linguistic rate. The S-FLM was evaluated by applying three financing scenarios to an airport infrastructure system (pessimistic, moderate/base, optimistic), where it was possible to show the impact of different linguistic rates on the EIRR. The behaviour of the S-FLM was validated using three different models: (1) a financial management tool; (2) a general FCM without pre-loaded causalities among the variables; and (3) a Statistical S-FLM model (S-FLMS), where the causalities between the concepts or rates were obtained as a result of an independent effects analysis applying a cross modelling between variables and by using a statistical multi-linear model (statistical significance level) and a multi-linear neural model (MADALINE). The results achieved by the S-FLM show a higher EIRR than expected for each scenario. This was possible due to the incorporation of an adaptive multi-linear causality matrix and a fuzzy credibility matrix into its structure. This allowed to stabilize the effects of the financial variables or rates on the EIRR throughout a financing period. Thus, the S-FLM can be considered as a tool to model dynamic financial scenarios in different knowledge areas in a comprehensive manner. This way, overcoming the limitations imposed by the traditional computational models used to design these financial scenarios.
\end{abstract}

Keywords: Financial modelling, Stochastic modelling, Fuzzy Cognitive Map, Logistic activation function, Financial Scenarios, Infrastructure Project finance.

\footnotetext{
${ }^{*}$ Corresponding author

Email addresses: jdgonza3@unal.edu.co (Juan David Gonzalez-Ruiz), pfjapena@gmail.com (Alejandro Peña ), e.duque@pascualbravo.edu.co (Eduardo Alexander Duque), alepaper@gmail.com (Alejandro Patiño), chiclana@dmu.ac.uk (Francisco Chiclana), mgongora@dmu.ac.uk (Mario Góngora)
} 


\title{
Stochastic logistic fuzzy maps for the construction of integrated multirates scenarios in the financing of infrastructure projects
}

\author{
Juan David Gonzalez-Ruiz ${ }^{\mathrm{a}, *}$, Alejandro Peña ${ }^{\mathrm{b}}$, Eduardo Alexander Duque ${ }^{\mathrm{c}}$, Alejandro Patiño ${ }^{\mathrm{b}}$, \\ Francisco Chiclana ${ }^{\mathrm{d}, \mathrm{e}}$, Mario Góngora ${ }^{\mathrm{d}}$ \\ ${ }^{a}$ Finance and Sustainability Reserch Group, Departamento de Economía, Universidad Nacional de Colombia, Medellín, \\ Colombia \\ ${ }^{b}$ Computational Intelligence and Automation Research Group, EIA University, Envigado, Antioquia, Colombia \\ ${ }^{c}$ Electronics Sciences 8 Informatics Research Group, Institución Universitaria Pascual Bravo, Medellín, Colombia \\ ${ }^{d}$ Institute of Artificial Intelligence (IAI), DeMontfort University, Leicester, UK \\ ${ }^{e}$ Department of Computer Science $\mathcal{G}$ Artificial Intelligence, University of Granada, Spain
}

\begin{abstract}
In general, the development of economic infrastructure systems requires a behavioural comprehensive analysis of different financial variables or rates to establish its long-term success with regards to the Equity Internal Rate of Return (EIRR) expectation. For this reason, several financial organizations have developed economic scenarios supported by computational techniques and models to identify the evolution of these financial rates. However, these models and techniques have shown a series of limitations with regard to the financial management process and its impact on EIRR over time. To address these limitations in an inclusive way, researchers have developed different approaches and methodologies focused on the development of financial models using stochastic simulation methods and computational intelligence techniques. This paper proposes a Stochastic Fuzzy Logistic Model (S-FLM) inspired by a Fuzzy Cognitive Map (FCM) structure to model financial scenarios. Where the input consists in financial rates that are characterized as linguistic rates through a series of adaptive logistic functions. The stochastic process that explains the behaviour of the financial rates over time and their partial effects on EIRR is based on a Monte Carlo sampling process carried out on the fuzzy sets that characterize each linguistic rate. The S-FLM was evaluated by applying three financing scenarios to an airport infrastructure system (pessimistic, moderate/base, optimistic), where it was possible to show the impact of different linguistic rates on the EIRR. The behaviour of the S-FLM was validated using three different models: (1) a financial management tool; (2) a general FCM without pre-loaded causalities among the variables; and (3) a Statistical S-FLM model (S-FLMS), where the causalities between the concepts or rates were obtained as a result of an independent effects analysis applying a cross modelling between variables and by using a statistical multi-linear model (statistical significance level) and a multi-linear neural model (MADALINE). The results achieved by the S-FLM show a higher EIRR than expected for each scenario. This was possible due to the incorporation of an adaptive multi-linear causality matrix and a fuzzy credibility matrix into its structure. This allowed to stabilize the effects of the financial variables or rates on the EIRR throughout a financing period. Thus, the S-FLM can be considered as a tool to model dynamic financial scenarios in different knowledge areas in a comprehensive manner. This way, overcoming the limitations imposed by the traditional computational models used to design these financial scenarios.
\end{abstract}

Keywords: Financial modelling, Stochastic modelling, Fuzzy Cognitive Map, Logistic activation function, Financial Scenarios, Infrastructure Project finance.

\footnotetext{
${ }^{*}$ Corresponding author

Email addresses: jdgonza3@unal.edu.co (Juan David Gonzalez-Ruiz), pfjapena@gmail.com (Alejandro Peña ), e.duque@pascualbravo.edu.co (Eduardo Alexander Duque), alepaper@gmail.com (Alejandro Patiño), chiclana@dmu.ac.uk (Francisco Chiclana), mgongora@dmu.ac.uk (Mario Góngora)
} 


\section{Introduction}

The Basel Committee has identified the financial performance, as one of the most important information categories to achieve adequate financial transparency. That is why financial performance should be addressed and clear terms and with an appropriate level of detail. The other categories are the financial position, risk management strategies and practices, risk exposures, accounting policies, basic business and corporate governance information [1,2]. Due to the dynamics of both, financial markets and business models, the creation of flexible financial models has become one of the main challenges for investments $[3,4,5]$. This is also true with regard to the constant development of social and economic infrastructure systems in Latin America in recent years $[6,7,8]$. In this context, infrastructure systems financing involves different financial variables or rates, analyzing them plays a pivotal role for the financial decision-making process. Similarly, the creation of financial scenarios that describe holistic situations in multiple aspects of investment infrastructure systems $[9,10]$. In this regard, the stochastic behaviour of financial variables, the relations among them and their evolution towards different equilibrium points represent a challenge when evaluating the success and financial performance of projects of this type $[11,12]$.

To understand a project's success according to the Equity Internal Rate of Return (EIRR), we proposed a model inspired by a traditional Fuzzy Cognitive Map (FCM), integrating in a single structure (i) the automatic characterization and sampling of the financial variables or rates, which are expressed as linguistic random variables; (ii) the interrelation between them; and (iii) their effect on the EIRR after executing a dynamic process, which is defined by the model. This integration provides a novel stochastic fuzzy logistic model (S-FLM), which contributes to make a difference with respect to the traditional FCMs and multi-variable financing models in three fundamental aspects: (i) it proposes a qualitative approach to financial planning at the strategic level, minimizing the utilization of crisp arithmetic values; (ii) it includes a fuzzy model structure that helps to overcome the problem of detailed numerical and/or statistical calculations that usually complicate the communication in the decision making process at the strategic-level decisions; and (iii) the sampling process for the linguistic random variables increases the understanding of the EIRR concept and requires less numerical analyses and more qualitative answers $[13,14]$.

For the analysis and validation of the S-FLM model, this paper proposes the creation of three investment scenarios (optimistic, moderate/base, pessimistic) for the financing of an airport infrastructure system. For this purpose, a total of six rates or financial variables were considered, which describe the typical business operations of an airport: the Growth Rate of Landings and Take-offs (GRLT); the Growth Rate of Airport Revenue (GRAR); the Growth Rate of Non-Airport Revenue (GRNAR); the Lease Growth Rate (LGR); the Growth Rate of Administration Costs (GRAC); and the Growth Rate of Costs of Operation and Maintenance (GRCOM). The input rates and the EIRR were automatically described as linguistic rates using an adaptive neural model with a generalized logistic activation function (NLAF) based on an auto-encoder learning process [15]. The causalities between the concepts or rates were obtained as a result of an independent effects analysis applying a cross modelling between variables [16] and by using a statistical multi-linear model (statistical significance level) and a multi-linear neural model (MADALINE). The integration of three processes for adaptation and learning in the structure of the S-FLM leads to a dynamic S-FLM model of reference for the creation of scenarios. These scenarios, model the financing of airport infrastructure projects but can also be used for the financing of infrastructure systems in general, where the integration of multiple rates or financial variables is required. The results that were achieved by the model for each of the aforementioned scenarios, show the stability of the estimation of the EIRR, according to the equilibrium process determined by the dynamic structure of the proposed S-FLM. The results show coherence with the financial performance, the rates and the financial variables that describe each of the proposed scenarios for this study.

The rest of the paper is structured as follows. In Section 2 previous work is summarized. In Section 3 we describe the methodology used to develop the proposed S-FLM model and its application in a case study. Section 4 presents an analysis of the results obtained by the S-FLM model in the learning and analysis stages. Conclusions and future lines of research are presented in Section 5. 


\section{Previous work}

In financial modelling, the scenario analysis is a process of examining and evaluating possible events that may take place in the future. This process is typically used to estimate changes in the value of a business or cash flow, especially when there are potentially favourable and unfavourable events that could affect a financial institution or project finance [17]. In this context, three well-defined research development trends can be identified in the scientific literature.

A first research development trend focuses on a series of aspects that are fundamental for the design of scenarios: data integration, sustainability, stochastic behaviour of parameters and variables, revenue, expenditures and profitability [17]. The sparsity of financial models that incorporate sustainability analysis in an environment of global change is highlighted by [18]. These authors contrast current global flows in the financial system with the future economic costs of a worldwide transition to renewable energies with the aim of achieving effective global ecological and financial sustainability. [19] describes the behaviour of non-arbitrage bounds using three financial scenarios: arbitrage opportunities will never exist, arbitrage may be present, and arbitrage opportunities will always exist. Using a Suppes-Bayes causal network, [20] carry out a series of simulations of financial stress test scenarios with the aim at discovering complex relations among the financial factors that affect the value of a portfolio. Within this research trend, [21] investigate the use of internal and external data obtained from stock markets to create financial scenarios to offer trading opportunities to investors. Financial scenarios that use a joint distribution of losses for assessing the stability of the banking sector in the EU, based on the Basel II capital rules and micro-simulations of portfolio structures, has been researched in [22]. [23] focus on the development of scenarios using stochastic and deterministic information to describe the financial fluctuations of a set of 345 stocks listed in the SP500 index that allow, in a general way, identifying the behaviour of a stock market. This trend is characterized by the application of financial models in different areas of knowledge and the integration of aspects that are important to describe the environment of an organization. However, there is a scarcity of financial models that integrate aspects of sustainability, stochastic behaviours, qualitative aspects of the operations of a business, and adaptation and learning processes that allow their automatic configuration based on data obtained through observation of systems and processes.

A second research development trend about the modelling of scenarios in different areas of knowledge and the forecasting and prediction of physical variables based on adaptation and learning models. In particular in the energy and the financial sectors. For instance, [24] apply different forecasting tools for the modelling of scenarios for universal access to renewable energy services in 2030 in sub-Saharan Africa. [25] study the development of scenarios to evaluate the demand of energy in real time according to the fluctuation of its supply. The simulation of the behaviour of electricity markets using multi-agent methodologies is investigated by [26]. While [27] propose back-casting models to create future scenarios derived from past energy collapse events. In this same trend line, the development of dynamic scenarios for financial analysis has also been dealt with using stochastic programming and traditional simulation techniques [28, 29]. Copulas were used to model nonlinear relations between the variables of a scenario as described in [30]. [19] created models to discriminate different scenarios according to their characteristics. In [31], the authors developed scenarios to evaluate the financial stability of the markets through the use of stress scenarios generated by accidental events in business operations. Within this research trend, the development of scenarios using neural networks for forecasting energy consumption according to the thermal comfort required by a house owner was addressed by [32]. A self-organized Neuro-Fuzzy System was proposed to model the dynamics and to forecast air cargo and airline passenger traffic in [33]. In addition, the integration of neural networks with cognitive maps for the analysis of scenarios has been proposed in a study of the dynamics of inflation in Turkey [34]. Stochastic fuzzy neural models that allow creating of dynamic scenarios, where the inputs are defined in terms of stochastic processes, also belong to this research development trend [35]. All these research studies clearly emphasize the relevance of different computational intelligence (CI) and simulation techniques in forecast modelling by using of scenarios. The studies include the energy sector and assessments of the stability of the financial sector. However, in this trend, the modelling and the relationships between variables using adaptive and learning models still missing. It includes models that identify points of equilibrium in variables that possess highly qualitative information or 
that have stochastic behaviours.

A third research development trend informs about the evolution that FCMs have experienced in the modelling of complex dynamic systems and the construction of scenarios to describe events and situations that might occur in the future. At an early stage of this research trend, [36] proposed a combined Delphi method, a FCM and a multi-criteria technique TOPSIS model to facilitate policy makers a tool for measuring the impact of a decision. The importance of FCM in realistic and measurable objectives in strategic planning projects, through a series of FCM sample maps with causal relationships, to generate financial performance indicators has been discussed by [1]. Whereas [37] showed, how the FCMs can be used to create futuristic data-driven scenarios incorporating text mining and fuzzy association rules with the objective to improve the sustainability of electric vehicles. How the FCMs can improve the intellectual capital (IC) in organizations by integrating the dimensions that define the IC concept was investigated by [38]. [39] presented an OCAM model (Online Cognitive Automated Mapper) based on an FCM to support the decision making in organizations without turning to experts. This was done by integrating information obtained from different sources in aspects such as: global crisis, the state of global economy, or technological development concepts. A second stage of development of this research trend is characterized by the future of decision making in different areas of knowledge by applying FCMs and integrating random variables. In this context, [40] presented a step-wise framework for modelling scenarios using FCM, as well as a discussion of the future research direction for FCM in different knowledge areas. [41] focused on a new conception of FCMs where it is established that, in addition to the values for the initial concepts. A series of values or disturbances are required in order to evaluate the stability of the FCMs in the representation of a system. In the field of medicine, [42] highlights four key areas for future research studies using different FCM structures: decision-making, diagnosis, prediction, and classification. With respect to the handling of incompleteness and natural uncertainty that characterize the connection matrix, a first proposal to improve the quality of an FCM where there is not enough data known as Interactive Evolutionary Computing (IEC) was put forward in [43]. This is a new optimization system based on partial expert estimations and evolutionary algorithms. Whereas [13] proposed a method combining FCMs and Bayesian Belief Networks(BBN) to improve the parameter setting capability of BBN in the modelling of operational risks. Within this development trend, we can clearly observe the importance of FCMs to support decision making and in the modelling of scenarios in different areas of knowledge where information is vague, fuzzy and incomplete. However, there is scarcity of models that allow for the automatic configuration of FCMs using random linguistic variables or that explain the evolution of the input variables according to the FCM structure. This gap can be noticed, especially in certain areas of knowledge, such as finance, where it is required to evaluate the evolution of rate and financial variables over time.

\section{Methodology}

The development of economics and financial-based scenarios can help to have a better understanding of how companies' daily operations reality. These scenarios can be influenced by multiple variables and aspects such as $[44,45,46]$ : projection of variables, financing rates, economic indicators, cash flows, and financing alternatives. At a tactical level, constructing financial scenarios requires a qualitative and quantitative description of these variables and aspects. Also, understanding the relationship between them, and integrating them in a single model [47]. Thus, the analysis and evaluation of these scenarios requires to take into account the associated complexity. In order to reduce complexity when analyzing financial multi-rate scenarios, this paper proposes a novel dynamic adaptive fuzzy integrated model that is inspired by the structure of an FCM, which is based on the methodology described below.

\subsection{Experimental study design}

For the analysis and evaluation of financial scenarios, the general scheme for financing an airport project by using a Project Finance (PF) methodology as developed by [7] and [48] will be used as a case study. PF refers to the structured financing of an economically independent entity created by sponsors, called Special Purpose Vehicle, using his own resources and debt. Its financing is based 
solely and exclusively on the cash flows generated by the project and the assets generated by the guarantee [9].

For the case study, the financing of an infrastructure is considered by applying an indebtedness scheme with the multilateral bank, combined with the investors' equity. The financial cost projection and the maximum debt amount are defined based on the free cash flow that is available for the explicit period of valuation. For the development of the model, the three scenarios mentioned before are considered. In this context, we determine the maximum (optimistic) and minimum (pessimistic) values of six variables associated with the airport infrastructure operation [48], as shown in Table 1.

Table 1: Critical Variables with Scenarios

\begin{tabular}{|c|c|c|c|}
\hline Critical Input Variables & Lower Limit & Case Base & Upper Limit \\
\hline Growth rate of landings and take offs (GRLT) & $10 \%$ & $12 \%$ & $15 \%$ \\
\hline Growth rate of airport revenue (GRAR) & $9 \%$ & $14 \%$ & $16 \%$ \\
\hline Growth rate of Non-Airport revenue (GRNAR) & $10 \%$ & $12 \%$ & $16 \%$ \\
\hline Lease growth rate (LGR) & $2 \%$ & $4 \%$ & $6 \%$ \\
\hline Growth rate of administration costs (GRAC) & $14 \%$ & $12 \%$ & $10 \%$ \\
\hline Growth rate of operation and management costs (GRCOM) & $16 \%$ & $14 \%$ & $9 \%$ \\
\hline
\end{tabular}

With respect to the complexity, traditional tools to build financial multi-variable scenarios present limitations to explain the behaviour of output variables such as the EIRR according to a deterministic behaviour presented by the input variables in different financial scenarios, which characterize these type of models as static models. For the design and development of financial scenarios using computational intelligence techniques, we proceed with the development of a reference database composed of a total of 1600 records by using the Scenario Manager tool of Microsoft Excel [48]. Each of the records is described according to the financing variables in Table 1, and its relationships with the output variable (EIRR). For the learning and set up phase of the proposed model, the normalization of the variables was carried out in the interval $[-1,1]$ and with the goal to describe the variables as linguistic rates using generalized logistic activation functions.

\subsection{Fuzzy characterization of financing rates}

The characterization of multi-rates financial scenarios based on fuzzy concepts is based on the characterization of the different rates as linguistic random variables, for which the following definitions are proposed [49]:

Definition 1. Random variables are variables that can take different values with different probabilities based on a probability distribution. Accordingly, a random variable can be represented by a cumulative distribution function $(\mathrm{CDF})$ through the following expression:

$$
F(x)=P(X \leq x)
$$

Definition 2. The generalized logistic distribution is a continuous probability distribution with a probability distribution function-pdf defined as follows:

$$
f(x, u, \sigma)=\frac{e^{-\left(\frac{x-u}{\sigma}\right)}}{\sigma\left(1+e^{-\left(\frac{x-u}{\sigma}\right)}\right)}
$$

where $x$ belongs to the domain of a random variable $X ; u$ is the distribution location factor $(u \in \mathbb{R})$; and $\sigma$ is a scaling factor that shows the dispersion of the investor's $\operatorname{EIRR}(\sigma>0)$.

The cumulative distribution function $c d f$ of a generalized logistic distribution can be expressed as

$$
F(x, u, \sigma)=\frac{1}{1+e^{-\left(\frac{x-u}{\sigma}\right)}}
$$


Definition 3. Given the quantiles that define a probability distribution $\left\{q_{o}, q_{1}, q_{2}, q_{3}, q_{4}\right\}$, and given a set of linguistic labels that describe qualitatively a random variable $\left\{c_{o}, c_{1}, c_{2}, c_{3}, c_{4}\right\}$, then the fuzzy sets characterizing the linguistic labels can be defined by the quantiles in terms of the structure of a cumulative distribution function (cdf) as follows:

Homogeneous fuzzy sets. When $\frac{1}{\sigma}=1$ and $u=0$, it configures a linguistic variable with homogeneous sets, where the quantiles are evenly distributed (Figure 1(a)).

Extended fuzzy sets. When $\frac{1}{\sigma}=2$ and $u=0$, it configures a linguistic variable with fuzzy sets defined by extended quantiles (Figure 1(b)).

Compressed fuzzy sets. When $\frac{1}{\sigma}=0.5$ and $u=0$, it configures a linguistic variable with fuzzy sets defined by compressed quantiles (Figure 1(c)).

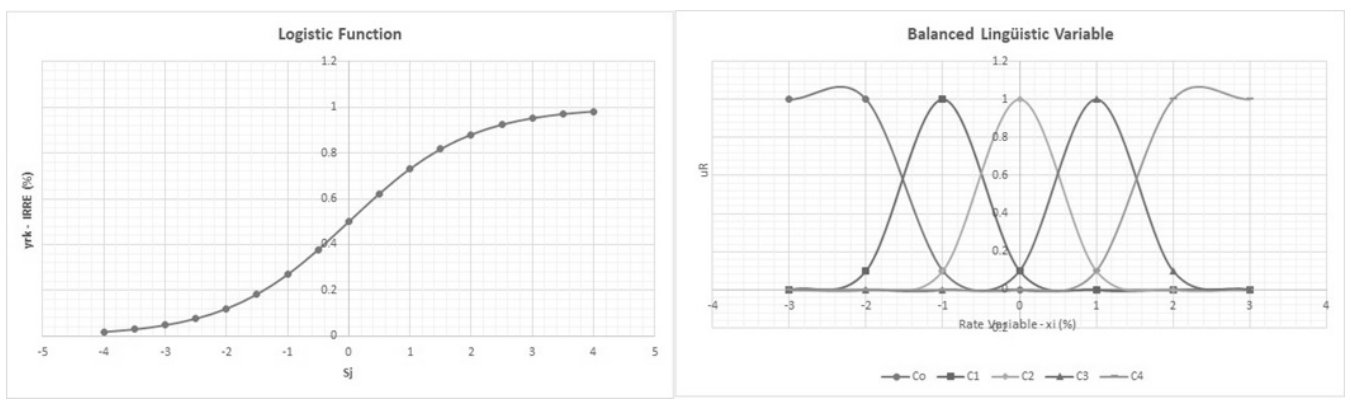

(a) Balanced Fuzzy Sets

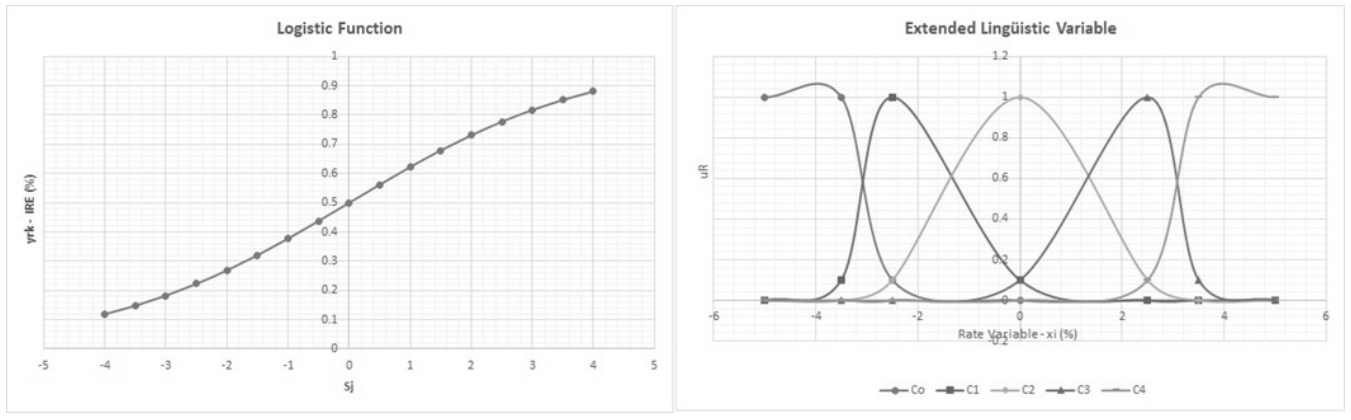

(b) Extended Fuzzy Sets

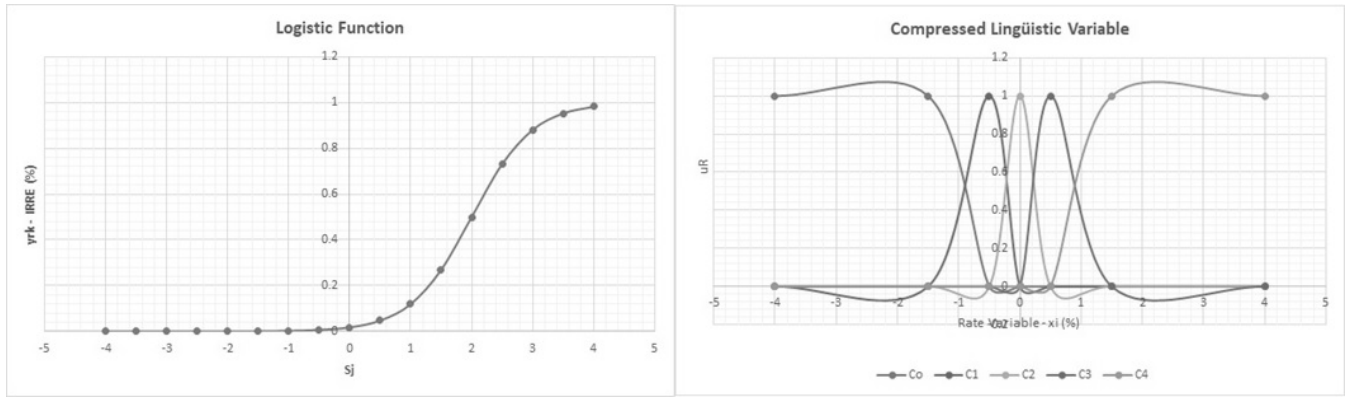

(c) Compressed Fuzzy Sets

Figure 1: Partial input linguistic rate

Definition 4. The generalized $c d f$ can be expressed by using the generalized logistic function in terms of its independent components as follows:

$$
F(x, \mu, \sigma)=\frac{1}{1+e^{-s_{j}}}
$$

where

$$
e^{-s_{j}}=e^{-\left(\frac{x_{1}-u_{1}}{\sigma_{1}}\right)} \ldots e^{-\left(\frac{x_{6}-u_{6}}{\sigma_{6}}\right)} \cdot e^{-a_{o} \cdot m}
$$




$$
e^{-s_{j}}=e^{-a_{o} \cdot m} \cdot e^{-a_{1}} \cdot e^{-a_{2}} \cdots e^{-a_{6}}
$$

and $e^{-a_{o} \cdot m}$ indicates the modified bias as a result of the transformation of the exponential term into a series of independent exponential terms, where $a_{i}$ indicates the partial effect of each of the variables $i(i=1,2,3,4,5,6)$ and $u_{i}$ indicates the variable values that form the base case according to Table 1 .

Definition 5 (Monte Carlo fuzzy sampling method). In accordance with the fuzzy sets obtained from a $c d f$, for the development of scenarios a new fuzzy sampling, which is based on the Monte Carlo method is proposed. This method has the following structure [49]:

$$
F S\left(p_{k}\right)=\sum_{j=1}^{n f s} u_{j}\left(p_{k}\right) \cdot F^{-1}\left(q_{j}\right)
$$

where $F S\left(p_{k}\right)$ is a fuzzy sampling function for a uniform random value $p_{k} \in[0,1] ; n f s$ is the number of fuzzy sets that conform a linguistic variable; $q_{j}$ is a uniform partitioning of the interval that defines $p_{k} ; F^{-1}\left(q_{j}\right)$ indicates the quantile function for $q_{j}$; and $u_{j}\left(p_{k}\right)$ is the (Gaussian) membership value of $p_{k}$ in the $j-f u z z y$ set:

$$
u_{j}(p k)=e^{-\frac{1}{2}\left(\frac{q_{j}-p_{k}}{D_{j}}\right)^{2}}
$$

with

$$
D j= \begin{cases}q_{j+1}-q_{j-1} & j<n f s \\ 2 \cdot\left(q_{j}-q_{j-1}\right) & j=n f s \\ 2 \cdot\left(q_{j+1}-q_{j}\right) & j=0\end{cases}
$$

The procedure for doing the sampling by using the Monte Carlo method on random linguistic variables is:

1. A random number $p_{k}$ is generated using a uniform distribution $p_{k} \in[0,1]$.

2. Computation of the membership degrees $u_{j}\left(p_{k}\right)$ with respect to the normalized fuzzy sets $j$ that represent a linguistic variable using (8).

3. Finally, the sampling value is obtained by using (9).

4. Steps 1,2 and 3 are repeated until a total of 1000 data points for each linguistic input variable is obtained.

\subsection{Neural stochastic model with partial activation logistic functions (NLAF)}

In order to identify the EIRR according to the input variables defined in Table 1, we propose a stochastic neural model with an logistic output function (NLAF), which is defined as follows:

$$
y r_{k}=\frac{1}{1+e^{\left(-\alpha \cdot s_{j}+\beta\right)}}
$$

where $y r_{k}$ indicates the output value for the EIRR that is estimated by the model for the reference record $k$ of the database, where $\alpha$ is a scale factor; $\beta$ is a translation factor and $s_{j}$ is the adaptive linear combiner, which is defined as:

$$
s_{j}=c_{1} \cdot h_{1}+c_{2} \cdot h_{2}+\ldots+c_{n o} \cdot h_{n o}
$$

Where no indicates the number of partial linear regressions or hidden neurons; $j$ indicates each hidden neuron or partial linear regression $(j=1,2, \ldots, n o) ; c_{j}$ represents the importance of each partial linear regression; and $h_{j}$ represents the $j$ partial linear regression:

$$
h_{j}=w_{j, o} \cdot x_{o}+w_{j, 1} \cdot x_{1}+\ldots+w_{j, n e} \cdot x_{n e}
$$


Where ne indicates the number of input variables; $w_{j i}$ is the support for each partial linear regression $(i=1,2, \ldots, n e)$; and $x_{i}$ represents the input variables.

The adaptive process is defined by the Generalized Delta Rule [50]:

$$
\begin{gathered}
c_{j}^{k+1}=c_{j}^{k}-\gamma \cdot \frac{\partial e_{k}^{2}}{\partial c_{j}^{k}} \\
w_{j i}^{k+1}=w_{j i}^{k}-\gamma \cdot \frac{\partial e_{k}^{2}}{\partial w_{j i}^{k}}
\end{gathered}
$$

Where $\gamma$ represents the learning factor; and $e_{k}^{2}$ is the root mean square error $(r s e)$ at instant $k$ :

$$
e_{k}^{2}=\frac{1}{2}\left(y d_{k}-y r_{k}\right)^{2}
$$

Where $y d_{k}$ representing the $E I R R$ obtained from multivariate tables using a Scenario Manager Tool of Microsoft Excel.

Based on (10), the learning rules take the following form:

$$
\begin{gathered}
c_{j}^{k+1}=c_{j}^{k}+\gamma \cdot \alpha \cdot e_{k} \cdot \frac{e^{\left(-\alpha . s_{j}+\beta\right)}}{\left(1+e^{\left(-\alpha \cdot s_{j}+\beta\right)}\right)^{2}} \cdot h_{j} \\
w_{j i}^{k+1}=w_{j i}^{k}+\gamma \cdot \alpha \cdot e_{k} \cdot \frac{e^{\left(-\alpha \cdot s_{j}+\beta\right)}}{\left(1+e^{\left(-\alpha . s_{j}+\beta\right)}\right)^{2}} \cdot c_{j} \cdot x_{i}
\end{gathered}
$$

According to Definition 4, and taking into account the propagation of each input variable through the structure, the decomposition of the model by independent effects is performed as follows:

$$
s j_{x i}=c_{1} \cdot w_{i, 1} \cdot x_{i}+c_{2} \cdot w_{2, i} \cdot x_{i}+\ldots+c_{n o} \cdot w_{n o, i} \cdot x_{i}
$$

Where $s j_{x i}$ indicates the effect of input variable $x_{i}$ on the consolidated output of the hidden layer or dot product between $x_{i}$ and $w_{j, i}$ for the partial regression $j$. According to equation (18), the dot product can be expressed:

$$
\begin{gathered}
s j_{x i}=\left(c_{j} \cdot w_{j i}\right) \cdot x_{i} \\
s j_{x i}=\sum_{i=1}^{n e} a i \cdot x_{i} \\
s j_{x i}=a_{o} \cdot x_{o}+a_{1} \cdot x_{1}+\ldots+a_{n e} \cdot x_{n e}
\end{gathered}
$$

Where $a_{i}$ indicates the independent's effects associated with the $i$ input variables. These variables are defined by the EIRR. According to Definition 3, the input variables can be expressed as partial linguistic rates in accordance with the logistic function using the independent effects expressed in (21). According to the normalization of the input variables in the interval $[-1,1]$, the independent effects takes a form based on (6).

\subsection{Stochastic Fuzzy Logistic Map (S_FLM)}

FCM is a modelling methodology for complex decision systems, which originated from the combination of Fuzzy Logic (FL) and Neural Networks (NN) [51]. A FCM describes the behaviour of a system in terms of concepts. Each concept represents an entity, a state, a variable or a characteristic of the system [56]. The structure of an FCM is defined as follows:

$$
C_{j}^{k+1}=f\left(C_{j}^{k}+\sum_{i=1}^{n e} C_{i}^{k} \cdot w m_{j, i}\right)
$$

Where $j$ indicates the number of variables, both the input and the output EIRR, so $j=1,2, \ldots, n e+$ 1; ne indicates the number of input variables; $C_{j}^{k}$ represents the value of the concept $j$ or the input 
linguistic rate $j$ at instant $k$. The EIRR is located in the $n e+1$ component; $w m_{j, i}$ represents the casual relationship, that exists between the linguistic input variable $i$ and the linguistic input variable $j$. When $w m_{j, i}>0$, a positive causality between the concepts exists. While $w m_{j, i}<0$ represents a negative causality; $f(\cdot)$ represents the logistic function that allows to support the normalization of the linguistic input variables in the interval $[-1,1]$ as shown through the following definition:

$$
f\left(x_{i}\right)=\frac{2}{1+e^{-x_{i}}}-1
$$

The iterative mechanism that is incorporated through FCM allows to describe the evolution of a system up to the points of equilibrium that are defined by the knowledge that is incorporated in the nodes and in their causal relationships. Unlike NNs, FCMs have a strong semantic that is defined by concepts. This characteristic makes them ideal for the representation of complex systems at a strategic level $[43,41]$. In this context, where the structure of the fuzzy sets defines the linguistic rates or nodes, the relations of causality between nodes can be expressed in terms of a fuzzy credibility model as proposed by [52]:

$$
w m c_{j, i}=e-\frac{1}{2}\left(\frac{u_{j, i 1}-u_{i, i 2}}{\sigma_{j, i 1}+\sigma_{i, i 2}}\right)
$$

Where $w m c_{j, i}$ indicates the credibility of relationships between the fuzzy sets that is representing the linguistic input variable $i$ and the fuzzy sets that is representing the linguistic input variable $j ; u_{j, i 1}$ and $u_{i, i 2}$ are the centres of the fuzzy sets $i 1$ and $i 2$ for the linguistic input rates $j$ and $i$, respectively; and $\sigma_{j, i 1}, \sigma_{i, i 2}$ represent the size of the fuzzy sets $i 1$ and $i 2$ for the linguistic input rates $j$ and $i$, respectively.

For the development of dynamic financial multi-variable scenarios that allow to describe the EIRR behaviour over time according to the FCM structure (22), a novel stochastic fuzzy logistic map (S_FLM) is proposed. This proposal allows to integrate in a single structure each of the aspects that define a financial scenario and the variables expressed as linguistic random rates, based on Definitions 3 and 5 , which can be expressed as follows [37, 53, 52]:

$$
C_{j}^{k+1}=f\left(C_{j}^{k}+\sum_{i=1}^{n e} C_{j}^{k} \cdot w m_{j, i} \cdot w m c_{j, i}\right) .
$$

In accordance with the structure of the NLAF function, the value that represents the EIRR is expressed as follows:

$$
C_{n e+1}^{k+1}=\frac{1}{1+e^{-C_{n e+1}^{k}}} .
$$

According to the S_FLM structure, the dynamic behaviour is given by an active learning process, which is integrated into the model to update causalities with respect to the variables. The S_FLM active learning process is denoted and defined as follows [50, 54]:

$$
\begin{gathered}
d w_{j, i}=\gamma_{2} \cdot d_{i 1} \cdot d_{i 2} \\
w m_{j, i}=w m_{j, i}+d w_{j, i} \\
d_{i_{1}}=C_{j, i 1}^{k+1}-C_{j, i 1}^{k} ; d_{i_{2}}=C_{i, i 2}^{k+1}-C_{i, i 2}^{k}
\end{gathered}
$$

Where $d_{i 1}, d_{i 2}$ indicates the change of the variables over time and in accordance with the cycles defined by the S_FLM model. Where $\gamma_{2}$ indicates the over-relaxation factor or dynamic learning factor.

In this context, the success of the financing of an infrastructure system is grounded on the equilibrium reached by the EIRR, according to the causal relationships among linguistic rates. This makes the FCM ideal to represent financial scenarios. In this context, the S_FLM represents a new approach to supplement a financial planning in infrastructure systems. Its provides a qualitative and intelligent support that is offered a strategy-level in order to shift focus from quantitative analysis towards an assessment at the strategy-level, according to the financial characteristics that define a financial scenario [51]. 


\subsection{Case study}

According to the structure of S_FLM, a financial case study scenario, which uses two input variables is proposed. One of them is associated to the operational growth which allows the financing of an airport project (GRLT). While a second variable is associated to the increase of the project's operating costs (GRCOM). The output variable is defined by the EIRR. For the development of this financial case study scenario, the following general methodology is proposed:

1. In this first moment, the financial base scenario is defined in Table 2 .

Table 2: Critical Variables with Scenarios

\begin{tabular}{|l|c|}
\hline Critical Variables & Base Case \\
\hline \hline Growth rate of landings and take-offs (GRLT) & $12.9 \%$ \\
\hline Growth rate of costs operation and maintenance (GRCOM) & $15.7 \%$ \\
\hline Equity Internal Rate of Return (EIRR) & $44.1 \%$ \\
\hline
\end{tabular}

2. To explain the rates as linguistic variables, an NLAF model is proposed applying three partial linear regressions $(n o=3)$. This way, the numeric structure of the NLAF model can be expressed at an initial stage as:

$$
w 2,3=\left[\begin{array}{lll}
-0.016 & 0.787 & -0.311 \\
-0.358 & 0.461 & -0.086 \\
-0.555 & 0.148 & -0.604
\end{array}\right], c 3=\left[\begin{array}{l}
0.315 \\
0.487 \\
0.110
\end{array}\right]
$$

3. The input vector is defined in accordance with Table 2 and the $y d_{k}$ that represents the expected EIRR, according to the input variables, can be expressed as follows:

$$
\begin{gathered}
\vec{x}=\left[\begin{array}{lll}
x_{o} & x_{G R L T} & x_{G R C O M}
\end{array}\right], y d=[E I R R] \\
\vec{x}=\left[\begin{array}{lll}
1 & 0.129 & 0.157
\end{array}\right], y d=[0.441]
\end{gathered}
$$

Taking into account that the input variables of the model were normalized in the interval $[-1,1]$; the GRLT and GRCOM variables can be expressed as:

$$
\vec{x}=\left[\begin{array}{lll}
1 & 0.556 & 0.954
\end{array}\right], y d=[0.441]
$$

4. The partial linear relations $h_{j}$ can be estimated in accordance with the $w_{j, i}$ matrix can be estimated:

$$
\begin{gathered}
h_{1}=1 \cdot(-0.016)+0.556 \cdot 0.787+0.954 \cdot(-0.311) \\
h_{2}=1 \cdot(-0.358)+0.556 \cdot 0.461+0.954 \cdot(-0.086) \\
h_{3}=1 \cdot(-0.555)+0.556 \cdot 0.148+0.954 \cdot(-0.604) \\
\vec{h}=\left[\begin{array}{lll}
0.3430 & 0.102 & -0.169
\end{array}\right]
\end{gathered}
$$

5. The output $E I R R$ can be estimated as follows:

$$
\begin{gathered}
s_{j}=0.0343 \cdot 0.315+0.102 \cdot 0.487-0.169 \cdot 0.110=0.139 \\
y r_{k}=\frac{1}{1+e^{-0.139}}
\end{gathered}
$$

6. For updating the model's weights:

$$
e_{k}=\left(y d_{k}-y r_{k}\right)=(0.441-0.534)=-0.093
$$

The learning factor is defined as $\gamma=0.03$. For the $C_{j}^{\prime} s$ :

$$
C_{j}^{k+1}:\left[\begin{array}{l}
0.314 \\
0.486 \\
0.109
\end{array}\right]=\left[\begin{array}{c}
0.135 \\
0.487 \\
0.110
\end{array}\right]+0.03 \cdot(-0.093) \cdot 0.016 \cdot\left[\begin{array}{c}
0.343 \\
0.102 \\
-0.169
\end{array}\right]
$$


According to (17), $w_{j i}^{\prime} s$ data update is as follows:

$$
W=\left[\begin{array}{lll}
-0.016 & 0.786 & -0.310 \\
-0.357 & 0.460 & -0.086 \\
-0.554 & 0.147 & -0.604
\end{array}\right]
$$

7. The independent effects for each input variable as follows, based on the logistic function:

$$
s j=-0.239 \cdot x_{o}+0.487 \cdot x_{G R L T}-0.206 \cdot x_{G R C O M}
$$

8. According to the decomposition of the LAF function by its components:

$$
y r k=\frac{1}{1+e^{-0.239 \cdot x_{o}} \cdot e^{-0.487 \cdot\left(x_{G R L T}\right)} \cdot e^{0.206 \cdot\left(x_{G R C O M}\right)}}
$$

9. Taking into account the base case for the $x_{G R C O M}$ input variable, and also the independent effect of the $G R L T$ for the interval $[-1,1]$, the partial logistic function is defined as:

$$
\vec{Q}=[\text { very low low medium high very high }]
$$

Where the quantiles that allow the development of the fuzzy sets are defined for this function:

$$
\vec{Q}=\left[\begin{array}{lllll}
-0.052 & 0 & 0.483 & 1 & 1.01
\end{array}\right]
$$

According to the logistic function shown in Figure 2, it can be observed that the fuzzy sets have an extended behaviour. That is due to the separation between quantiles. That gives a soft logistic function. In this way, the base case scenario can be described qualitatively in terms of the linguistic variables that define an input variable of the model as shown in Table 3.

Table 3: Critical Variables of the Scenarios

\begin{tabular}{|l|c|c|c|}
\hline Critical Variables & Base Case & Normalized Base & Fuzzy Sets \\
\hline \hline GRLT & $12.9 \%$ & 0.449 & Medium \\
\hline GRCOM & $15.7 \%$ & 0.752 & Low \\
\hline
\end{tabular}

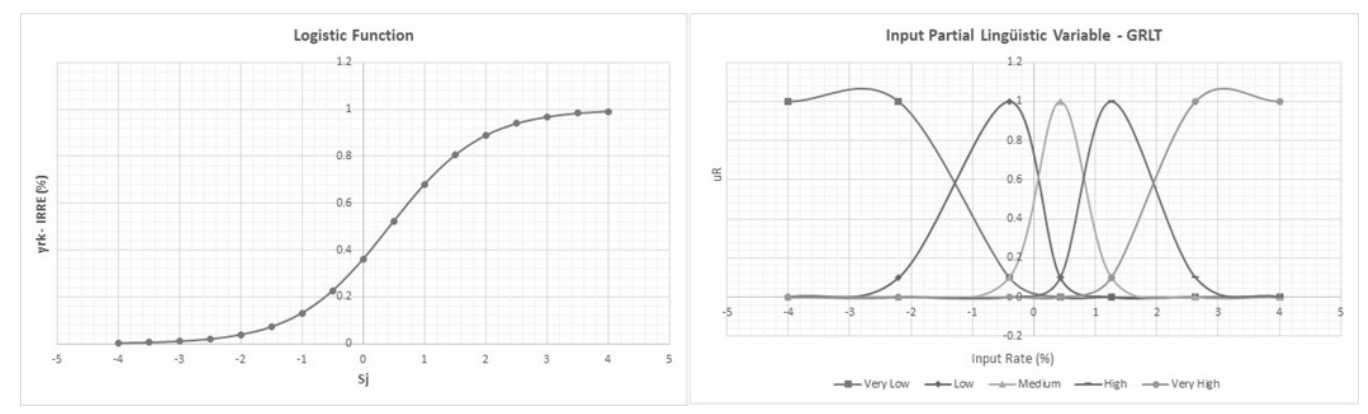

Figure 2: Partial logistic distribution of the GRLT effect

\subsection{Experimental Validation}

For the analysis and general validation of the proposed S_FLM model, four stages were considered. As a basis for the analysis, a data set composed of 1600 records that describe the EIRR in terms of six commercial rates (Table 1) was constructed. These records were obtained by using the Excel Scenarios Manager Tool. According to this data set, in a first stage we proceeded to identify the structure of EIRR for a reliability of a $99.9 \%$ using an NLAF (neural logistic adaptive function) model. This way, the learning process was executed for a total of 1000 tests, where each test is composed by a total of $i_{1}=500$ cycles of learning. The stop criteria for each test was defined by the rse (root mean square error) as follows: $\left\|r s e_{i_{1}+1}-r s e_{i_{1}}\right\|<5 \cdot 10^{-3}$, while the learning behaviour was evaluated by using the 
fuzzy model proposed by [55]. According to this model, it expect that the NLAF model reaches values close to zero for Fractional Bias (FB), Normalized Mean Square Error (NMSE), Unpaired Accuracy of Peak (UAPC2) and Mean Relative Error (MRE) indices. Furthermore, values close to one for the Geometric Mean (MG), Geometric Variance (VG), Index of Agreement (IOA) and FAC2 (Factor of Two) indices. The characterization of input rates as linguistic rates was carried out based on Definition 3 and Definition 4. Thus, it can be expected that GRLT, GRAR, GRNAR, and LGR achieve positive effects, while for the variables GRAC and GRCOM a negative effect is expected.

In a second stage, we proceeded with the design of the proposed model ( $\left.S_{-} F L M\right)$ based on three basic criteria: characterization of rates as linguistic rates (NLAF model), the causalities or partial relationships between fuzzy sets that characterize these linguistic variables, and the evolution of the EIRR over time. In order to established the causalities or partial relationships, an independent effects analysis by means of a cross modelling between linguistic variables using a statistical multilinear model, and a multi-linear neural model (MADALINE) was carried out [16]. The statistical significance, given by the statistical multi-linear technique for each effect, can be highlighted as it also gives validity to the causalities obtained by using a MADALINE model. The cross modelling process was evaluated using the index of agreement IOA for both models, while the MADALINE learning process was carried out for the aforementioned data set and for a total of $i_{1}=200$ learning cycles, where the stop criteria was defined by the rse error.

In a third stage, the structure analysis for the proposed $S_{-} F L M$ model was carried out by using three different financing scenarios: a Pessimistic Scenario, a Base Case or Moderate Scenario, and an Optimistic Scenario. Each scenario is qualitatively described in terms of the fuzzy sets that defines each linguistic input and output rates. For each scenario, we established an expected EIRR reference based on a sub-dataset (Table 2). Also, a reference value for each input linguistic rate. In this stage, it is expected that the linguistic variables with extended fuzzy sets or positive effects have a greater positive impact on the EIRR, as opposed to the compressed linguistic variables or negative effects, which have a discrete effect on the EIRR.

In a fourth stage, the validation of the proposed $S_{-} F L M$ model was carried out by using three models for each aforementioned scenarios: A first model is based on an FCM structure, a second model based on an $S_{-} F L M$ with statistical causalities structure $\left(S_{-} F L M S\right)$ and a third model based on an $S \_F L M$ with an adaptive multilinear causalities structure $\left(S \_F L M M\right)$. To overcome the limitations exposed by traditional models in constructing dynamic financial scenarios, each model was executed for a total of 50 cycles or financing periods (evolution process), and submitted to a sampling process for the fuzzy sets that characterize each linguistic rate. This was done in order to assess its stability estimating the expected EIRR rate. The interval sampling for each linguistic input rate was defined by the maximum and minimum values of each linguistic input rate, according to the financial scenarios aforementioned. Here, the behaviour of the models was evaluated based on the index of agreement (IOA) for the input rates, before and after the evolution process, and according to the statistical variation of the expected EIRR for each scenario. Here, we expected an IOA close to $80 \%$, with EIRRs much higher than the expected EIRRs for each scenario, due to the integration of a credibility matrix into the $S_{\_} F L M M$ model which allows stabilizing the estimation of EIRR more quickly.

\section{Experimental results}

Figure 3 shows the behaviour of the NLAF model in the identification of the EIRR for one of the 1000 tests that were applied for the configuration of the model. Figure 3 highlights two parts or sub-sets of data used for the validation of the model in the first stage $(1600$ - records $)$ : a first part corresponding to $50 \%$ of the data $(800$ - recordsoflearning), which allows configuring the model by adaptation and learning. A second part, correspond to $50 \%$ (800-recordsofautonomy), which allows evaluating the autonomy of the model in the estimation of the EIRR without applying a learning process. According to the metrics established for the analysis and validation of the model, the results given by the NLAF model in the estimation of an EIRR of reference (Figure 3), show that the model achieved for both sub-data sets (learning and autonomy), IOAs above 90\% (0.96323, 0.93498). This was confirmed by a FAC2 near to one $(1.00000,0.98888)$, which indicates the good behaviour of the model in forecasting the EIRR. The statistical stability of the model is underlined by the MG 


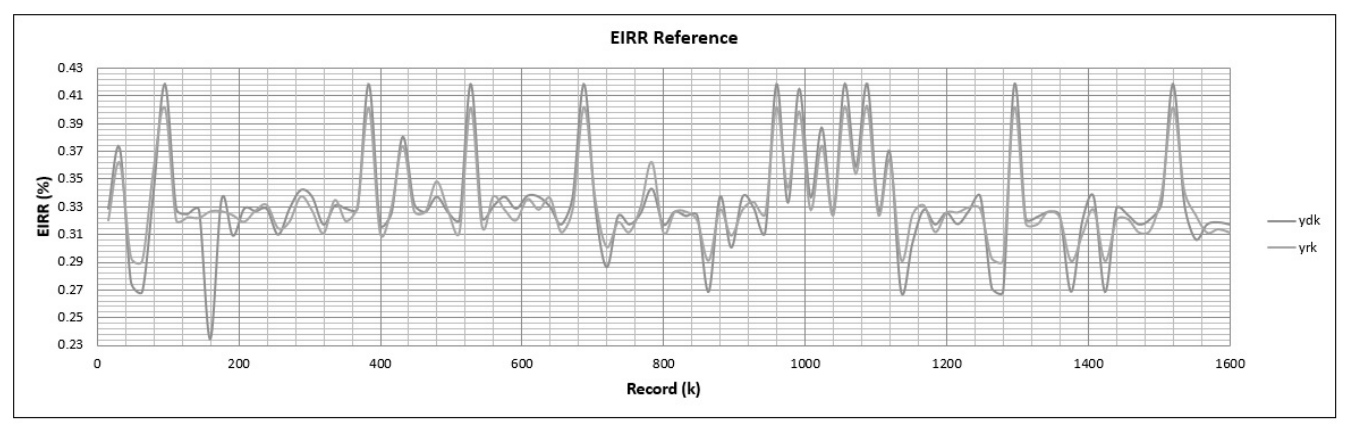

Figure 3: Results given by the model in the learning and configuration phase

(0.99976, 0.99888) and VG $(1.00090,1.00012)$, which obtained values near to one, indicating that the data possibly follow the same probability distribution. The behaviour of the error in the learning and validation process were evaluated by using the FB $(-7.5162 \mathrm{E}-05,-0.00097)$, the NMSE $(9.1698 \mathrm{E}-$ $05 ; 0.00012)$ and the $\operatorname{MRE}(-0.00030,-0.00119)$. Where the negative values indicate the trend of the NLAF model to sub-estimate the EIRR data of reference. In general, these indices show that the model behaves quite well in both, the adaptation and learning and also in the validation phase, in absence of a learning process. According to the structure of the activation function that defines the NLAF model, it becomes clear that the model succeeds in identifying the intrinsic structure of the data that represent the EIRR variable as evidenced by the values reached by the NMSE, VG, MG, IOA indices, depending on the rates that define a financial scenario.

The independent effects that characterize the EIRR rate based on Definition 4 (Table 4) show that the rate with the major partial positive effect is the GRLT with a value of 0.75661 related to the growth rate of landings and take-offs, while the GRAC rate related to the operational management costs achieved the major negative partial effect with a value of -0.58408 , which is in accordance with the definitions in this financial project. In general, the independent effects were coherent with the definition in this financial project. The statistical variance after a 1000 learning tests, shows on average a variation close to $5 \%$ for each independent effect, which shows the stability of the model in the estimation of the EIRR rate. This behaviour can be expressed by using a deployment of the input rates applying an independent analysis based on a general LAF function 4 . In Figure 4, it can be observed that the rates with a positive effect present a great deployment in the conformation of the EIRR rate as shown by the GRLT, GRAR, GRNAR and LGR rates, while the rates with negative effects present a compression of deployment in the conformation of the EIRR rate, as shown by the GRCOM and GRAC rates, when these rates have an independent increase. The structural stability, achieved by the model can be seen through the logistic functions that represent each of the variables that configure a financial scenario. Also, through its effect on the IERR. This property makes the NLAF model ideal for characterizing the rates of a financial scenario, in terms of the structure of the IERR.

Table 4: Neural logistic independent effects

\begin{tabular}{|l|c|c|c|c|c|}
\hline Critical Variables & $a_{i}$ & Base Case $u_{i}$ & Variance & St.Dev. & Variation (\%) \\
\hline \hline$x_{o}$ & -0.42331 & 0 & 0.000586 & 0.024219 & 5.721 \\
\hline GRLT & 0.756607 & 0.42857 & 0.000894 & 0.029916 & 3.953 \\
\hline GRAR & 0.020119 & 0.71428 & $1.454 \mathrm{E}-06$ & 0.001206 & 5.994 \\
\hline GRNAR & 0.05129 & 0.42857 & $4.076 \mathrm{E}-06$ & 0.002019 & 3.936 \\
\hline LGR & 0.26894 & -0.71428 & 0.000182 & 0.013517 & 5.026 \\
\hline GRAC & -0.58408 & 0.42857 & 0.000856 & 0.02927 & 5.011 \\
\hline GRCOM & -0.24355 & 0.71428 & 0.000175 & 0.013252 & 5.441 \\
\hline
\end{tabular}

In Figure 5 the independent logistic behaviour per input rate can be observed, where the independent rates with major changes were the GRAR positive rate and the GRCOM negative rate. In Figure 


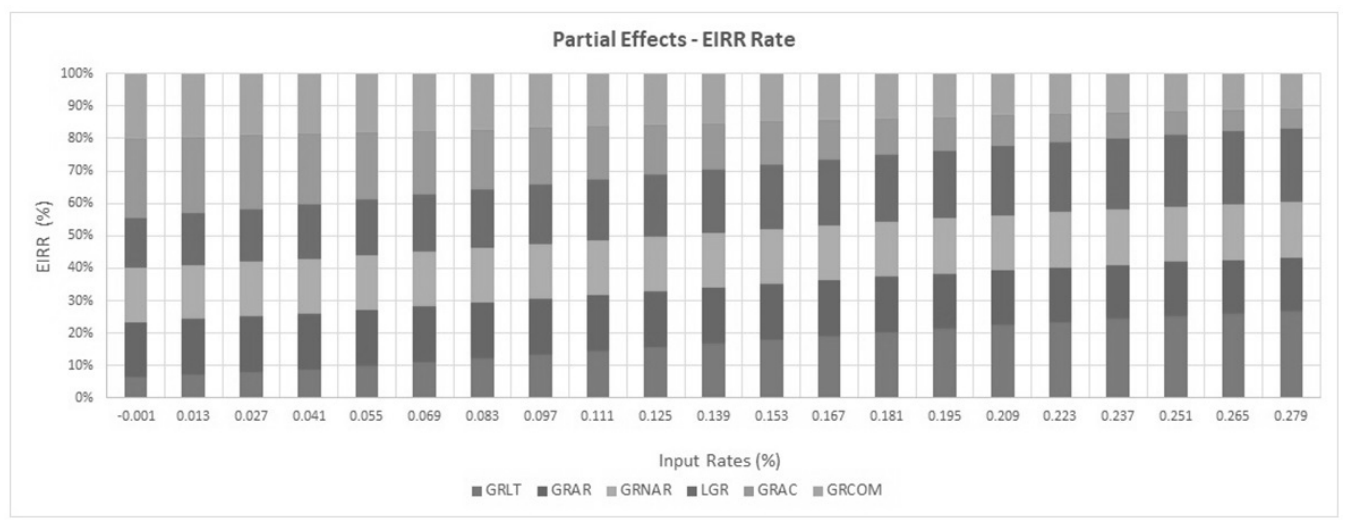

Figure 4: Diagram of Partial effects diagram of each of the rates on the EIRR

6 , the structure and shape of the fuzzy sets obtained, based on Definition 5 from the NLAF-LGR and the NLFA-GRLT rate respectively, can be observed. Here, the soft slope of the LAF functions exhibit more extended fuzzy sets with a greater impact on the output variable because the quantiles are located in a larger interval, as it happens with the GRLT rate, unlike the logistic functions with strong changes, as it happens with the LGR rate. This leads to compressed fuzzy sets, where the quantiles located in a much shorter interval generate a discrete impact on the EIRR or output variable. The compressed and uncompressed behaviour of the fuzzy sets is in accordance with the behaviour exhibited by the rates that are shown in Figure 6 .

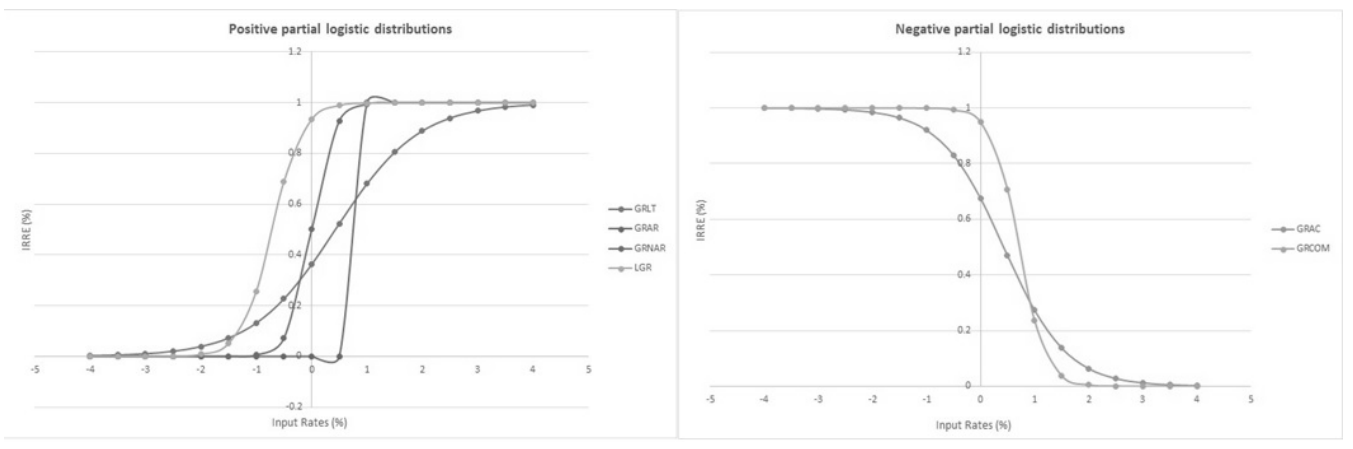

Figure 5: Partial logistic functions that represent the input variables or financial rates - Negative partial logistic distribution
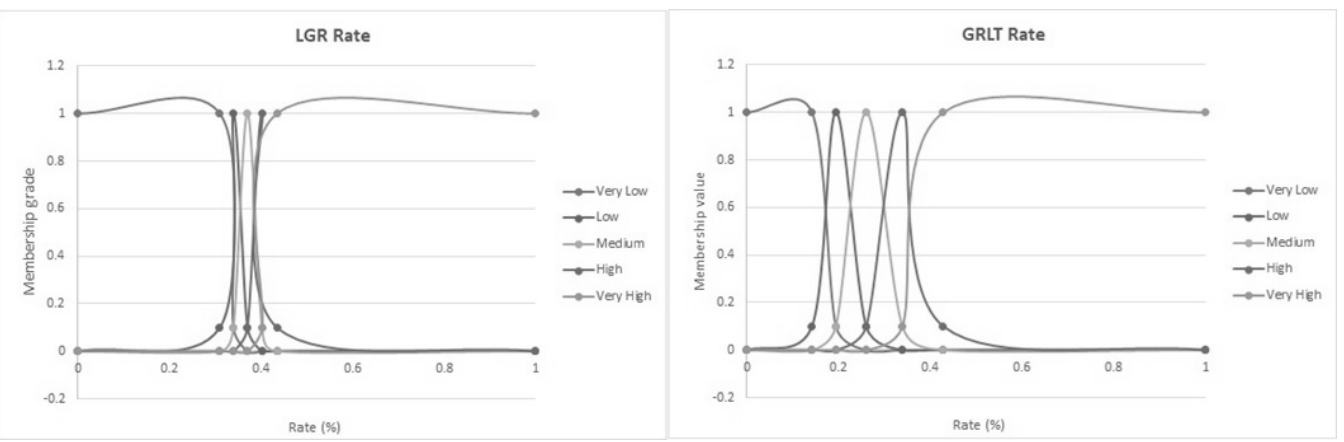

Figure 6: Fuzzy sets obtained from partial logistic functions - Extended Fuzzy sets GRLT

According to the structure and shape of the fuzzy sets that define a linguistic rate, Table 5 shows a qualitative description of the financial scenarios as defined in Table 1. This qualitative description refers to the logistic functions with smooth behaviours, as presented in the GRCOM and LGR rates. These used fewer qualities to describe the financial scenarios, unlike to the GRAR and GRAC, which presented pronounced logistic functions that required a greater number of fuzzy sets to express these same scenarios. This variety of fuzzy sets generated a significant effect of change with respect to the 
EIRR.

Table 5: Qualitative description of financial scenarios

\begin{tabular}{|l|c|c|c|c|c|c|}
\hline Critical Variables & Pessimistic & Fuzzy Set & Base Case & Fuzzy Set & Optimistic & Fuzzy Set \\
\hline \hline GRLT & $10 \%$ & Medium & $12 \%$ & Medium & $15 \%$ & Very High \\
\hline GRAR & $9 \%$ & Low & $14 \%$ & High & $16 \%$ & Very High \\
\hline GRNAR & $10 \%$ & Medium & $12 \%$ & Medium & $16 \%$ & Very High \\
\hline LGR & $2 \%$ & Very Low & $4 \%$ & Very Low & $6 \%$ & Medium \\
\hline GRAC & $14 \%$ & Very High & $12 \%$ & High & $10 \%$ & Medium \\
\hline GRCOM & $16 \%$ & Very High & $14 \%$ & Very High & $9 \%$ & Low \\
\hline
\end{tabular}

In the second stage, and in accordance to the general structure of the proposed model (S_FLM), we can observed in Table 6 and in Table 7 the causal relationships among the linguistic variables that describe the EIRR. These relationships were obtained as a result of an independent effects analysis by means of a cross modelling process with regard to the rates by using a statistical multi-linear model, and an adaptive multi-linear neural model (MADALINE). On the main diagonal of the tables (bold letters), we can observed the IOAs that were reached by the proposed models after the cross modelling process for each variable. In general it can be said that the IOAs achieved when modelling the variables GRTL, GRAR and GRAC were favourable for both models. It is also important to highlight the degree of statistical significance that the rates achieved with regard to the EIRR, as evidenced by the stars located below each variable in Table 6. Additionally, it stands out the high magnitude of the causal relationships that were reached by these variables when applying the MADALINE model in modelling the output IERR rate. This shows the importance of these variables for the financing of this type of infrastructure projects, as well as the stability of the proposed models in relation to the modelling of the EIRR intrinsic structure.

Table 6: Causal relationships among the linguistic variables - Independent effects analysis - Statistical multi-linear model

\begin{tabular}{|c|c|c|c|c|c|c|c|c|}
\hline & Bias & GRLT & GRAR & GRNAR & LGR & GRAC & GRCOM & EIRR \\
\hline \hline GRLT & -0.14425 & $\mathbf{0 . 2 1 2 5 8}$ & -0.20498 & -0.03933 & -0.07224 & 0.65701 & 0.15270 & 1.01081 \\
\hline & $* * *$ & & $* * *$ & $*$ & $* *$ & $* * *$ & $* * *$ & $* * *$ \\
\hline GRAR & 0.07384 & -1.48026 & $\mathbf{0 . 3 3 3 1 1}$ & -0.0606 & -0.11293 & 1.02786 & 0.23887 & 1.58004 \\
\hline & & $* * *$ & & & & $* * *$ & $* *$ & $* * *$ \\
\hline GRNAR & -0.28185 & -0.8813 & -0.18803 & $\mathbf{0 . 0 0 6 2 9}$ & -0.05919 & 0.44454 & 0.10504 & 0.82822 \\
\hline & $* *$ & $*$ & & & & & & $*$ \\
\hline LGR & -0.0949 & -0.61878 & -0.13394 & -0.02263 & $\mathbf{3 . 3 4 8 E - 0 5}$ & 0.43450 & 0.10093 & 0.66374 \\
\hline & & $* *$ & & & & $*$ & & $* *$ \\
\hline GRAC & 0.21388 & 1.23146 & 0.26678 & 0.03719 & 0.09508 & $\mathbf{0 . 1 4 6 3 7}$ & -0.20398 & -1.33038 \\
\hline & $* * *$ & $* * *$ & $* * *$ & & $*$ & & $* * *$ & $* * *$ \\
\hline GRCOM & 0.54508 & 1.27896 & 0.27705 & 0.03927 & 0.09870 & -0.91151 & $\mathbf{0 . 0 0 0 2 6}$ & -1.38092 \\
\hline & $* * *$ & $* * *$ & $* *$ & & & $* * *$ & & $* * *$ \\
\hline EIRR & 0.14297 & 0.93226 & 0.20179 & 0.03409 & 0.07147 & -0.65463 & -0.15206 & $\mathbf{0 . 9 2 8 7 7}$ \\
\hline & $* * *$ & $* * *$ & $* * *$ & $*$ & $* *$ & $* * *$ & $* * *$ & \\
\hline \hline
\end{tabular}

\begin{tabular}{|c|c|c|c|c|c|c|c|c|c|}
\hline \hline \multicolumn{10}{|c|}{ Significance Codes } \\
\hline \hline 0 & $* * *$ & 0.001 & $* *$ & 0.01 & $*$ & 0.05 &. & 0.1 & \\
\hline
\end{tabular}

In the third stage and according to the structure of the linguistic rates, Figure 7 shows the behaviour of the FCM, $S_{-} F L M S$ and $S_{-} F L M M$ in the modelling of dynamic financial scenarios after 50 financing periods or cycles. In general, it can observed that the models achieved a convergence with regards to the estimation of the EIRR, with IOA values on average close to $80 \%$ with respect to the structure of the rates for each scenario (Table 8). The observed higher EIRR obtained by the proposed S_FLMM model, was mainly due to the integration of the credibility matrix into the structure of the model. The integration generates convergence towards higher values with lower differences between the input 
Table 7: Causal relationships among the linguistic variables - Independent effects analysis - Adaptive multi-linear model (MADALINE)

\begin{tabular}{|c|c|c|c|c|c|c|c|}
\hline & GRLT & GRAR & GRNAR & LGR & GRAC & GRCOM & EIRR \\
\hline \hline GRLT & $\mathbf{0 . 5 8 2 5 4}$ & -0.10157 & -0.07587 & -0.05434 & 0.07870 & 0.00733 & 0.64715 \\
\hline GRAR & -1.63427 & $\mathbf{0 . 3 2 7 0 8}$ & -0.07869 & -0.12481 & 1.15400 & 0.32020 & 1.75016 \\
\hline GRNAR & -0.06160 & -0.18836 & $\mathbf{0 . 0 0 2 4 3}$ & -0.02529 & -0.10472 & -0.16870 & -0.01674 \\
\hline LGR & -0.01346 & 0.00328 & 0.00068 & $\mathbf{0 . 0 0 0 4 5}$ & -0.00077 & -0.00234 & 0.02446 \\
\hline GRAC & 1.21451 & 0.46497 & -0.00639 & 0.09230 & $\mathbf{0 . 1 3 3 1 1}$ & -0.00751 & -1.32045 \\
\hline GRCOM & -0.01460 & 0.58423 & -0.18028 & 0.00270 & 0.18457 & $\mathbf{2 . 9 9 E - 0 6}$ & -0.00051 \\
\hline EIRR & 0.8977 & 0.26548 & -0.00685 & 0.02700 & -0.57327 & 0.00268 & $\mathbf{0 . 8 9 9 7 8}$ \\
\hline
\end{tabular}

rates, as in the baseline scenario and the optimistic scenario. The EIRR value is in accordance with the magnitude of the rates that define each of the scenarios proposed for this study.

Table 8: Evolution of the EIRR over time

\begin{tabular}{|c|c|c|c|c|c|c|c|c|c|c|c|c|}
\hline Critical Variables & Pessimistic & FCM & $\begin{array}{r}\text { Id } \\
\text { S_FLMS }\end{array}$ & S_FLMM & Base Case & FCM & S_FLMS & S_FLMM & Optimistic & FCM & S_FLMS & S_FLMM \\
\hline GRLT & 0.10000 & $\begin{array}{c}0.14540 \\
\end{array}$ & 0.14570 & 0.09900 & 0.12000 & ב0.16200 & 0.14680 & 0.0999 & 0.14000 & 0.14640 & "0.14680 & "0.14610 \\
\hline GRAR & 0.09000 & 0.14680 & 0.14680 & 0.15510 & 0.11000 & 0.15000 & 0.15010 & 0.15510 & 0.13000 & 014940 & 0.15010 & 0.09490 \\
\hline GRNAR & 0.14000 & 0.10890 & 0.10930 & 0.11370 & 0.13000 & 0.10910 & 0.10930 & 0.11370 & 0.12000 & 0.10910 & 0.10930 & 0.14630 \\
\hline LGR & 0.02000 & 0.029300 & 0.02940 & 0.04380 & 0.04000 & 0.03000 & 0.03000 & 0.04620 & 0.06000 & 0.02990 & 0.03000 & 0.03630 \\
\hline GRAC & 0.14000 & 0.13160 & 0.13170 & 0.13850 & 0.12000 & 0.13690 & 0.13670 & 0.13850 & 0.10000 & 0.13720 & 0.13670 & 0.09650 \\
\hline GRCOM & 0.12000 & 0.15410 & 0.15400 & 0.4980 & 0.10000 & 0.15310 & 0.15380 & 0.14980 & 0.080000 & 0.15410 & 0.15380 & 0.10020 \\
\hline EIRR & 0.238641 & 0.24890 & 0.24970 & 0.25840 & 0.361836 & 0.24430 & 0.24550 & 0.37280 & 0.499268 & 0.24580 & 0.24550 & 0.52760 \\
\hline IOA & & 0.88921 & 0.88964 & 0.90272 & & 0.84543 & 0.86244 & 0.96547 & & 0.80298 & 0.80271 & 0.99101 \\
\hline
\end{tabular}

This fact corroborates the stability of the S_FLM in the estimation of EIRR over time. However, the FCM and $S_{-} F L M S$ converged at the same EIRR value for all scenarios, unlike the $S_{-} F L M M$ model, which achieved a higher EIRR than the expected EIRR, due to the capacity of the model to establish additional relationships based on the credibility matrix. These relationships represent the partial relationships among fuzzy sets, which describe the linguistic variables. As a consequence of this process, Table 9 shows the qualitative description for each scenario before and after the evolution process. The convergence towards higher EIRR values and the qualitative description of a scenario, makes the model suitable as an integral tool to model dynamic financial scenarios with multi-input rates.

Table 9: Qualitative description of the EIRR evolution over time

\begin{tabular}{|l|c|c|c|c|c|c|c|c|c|c|c|c|}
\hline Critical Variables & Pessimistic & FCM & S_FLMS & S_FLMM & Base Case & FCM & S_FLMS & S_FLMM & Optimistic & FCM & S_FLMS & S_FLMM \\
\hline \hline GRLT & Medium & High & High & Medium & Medium & High & High & Medium & Very High & High & High & High \\
\hline GRAR & Low & High & High & High & High & High & High & High & Very High & High & High & Medium \\
\hline GRNAR & Medium & High & High & High & Medium & High & High & High & Very High & High & High & High \\
\hline LGR & Very Low & Medium & Medium & Medium & Very Low & Medium & Medium & Medium & Medium & Medium & Medium & Medium \\
\hline GRAC & Very High & High & High & High & High & High & High & High & Medium & High & High & Medium \\
\hline GRCOM & Very High & High & High & High & Very High & High & High & High & Low & High & High & Medium \\
\hline
\end{tabular}

One of the main elements at the moment, when making a decision to invest in these type of projects, is related to the variation of the EIRR. For this reason, at the final stage, a sampling Monte Carlo process on S_FLMM was carried out by using Definition 5. This was done with the goal to characterize the EIRR variable as a probability distribution for each scenario. To achieve a reliability closed to $99.9 \%$, the sampling process was carried out for a total of 1000 records per linguistic input variable, where the sampling interval for each variable was defined by the distance between the values of the variables defined for each scenario, as shown in Table 10. The statistical properties for each variable are defined by the structure and shape of the fuzzy sets that characterize a linguistic rate described in [49].

Table 11 displays a set of distributions that allow modelling the EIRR as a probability distribution, as a result of the Monte Carlo sampling process carried out on the $S \_F L M M$ structure. Here, the EIRR can be expressed using six probability distributions. Among these distributions the following can be highlighted Normal, Cauchy and Logistic distributions. According to the Log-liklelihood and Kolmogorov Smirnov indices, the Normal distribution presents the best behaviour. However, the 
EIRR Rate Behavior (Pessimistic Case)

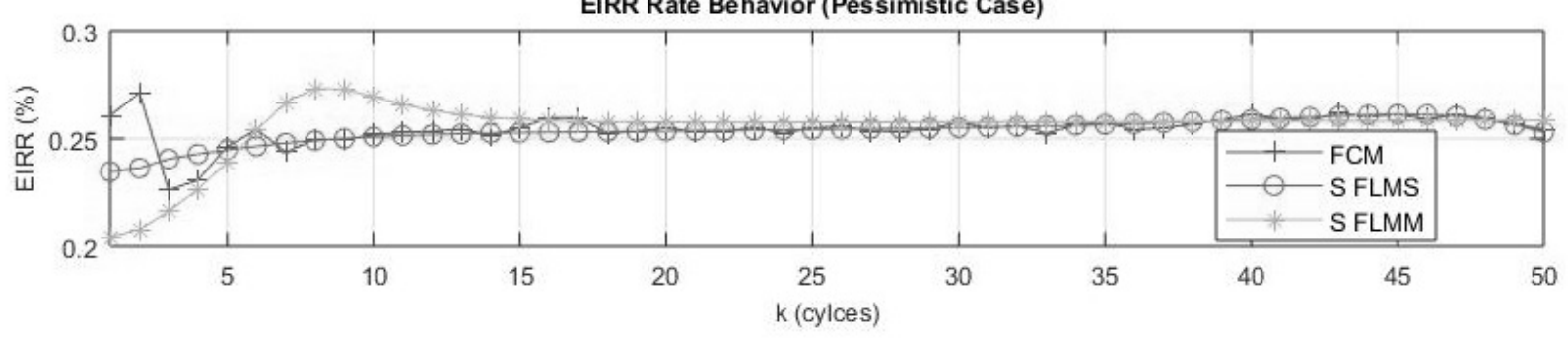

EIRR Rate Behavior (Base Case)

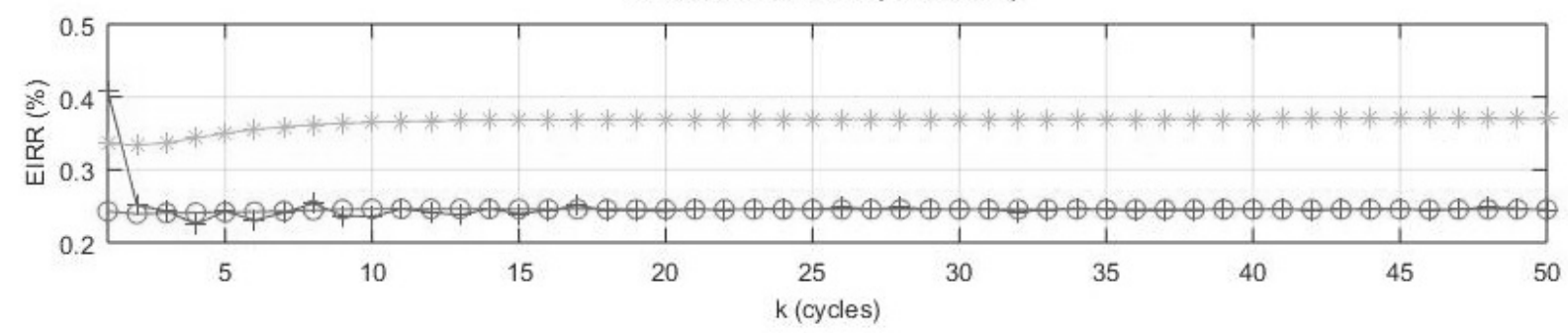

EIRR Rate Behavior (Optimistic Case)

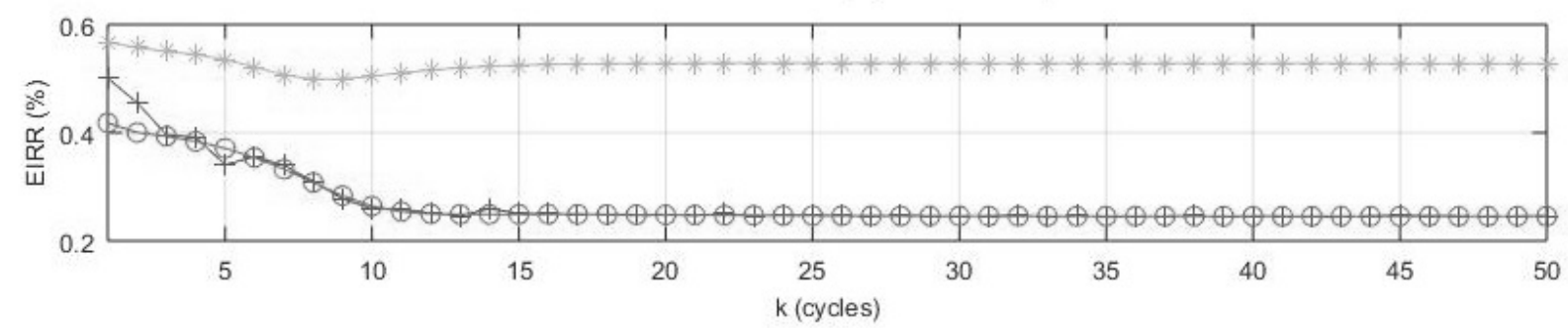

Figure 7: Evolution of the EIRR variable according to the financial scenarios.

Table 10: Sampling intervals for each of the scenarios

\begin{tabular}{|c|c|c|c|}
\hline Critical Variables & Pessimistic & Base Case & Optimistic \\
\hline \hline GRLT & {$[0.08000,0.120000]$} & {$[0.1000,0.14000]$} & {$[0.12000,0.16000]$} \\
\hline GRAR & {$[0.0700,0.11000]$} & {$[0.09000,0.13000]$} & {$[0.11000,0.15000]$} \\
\hline GRNAR & {$[0.15000,0.13000]$} & {$[0.14000,0.12000]$} & {$[0.13000,0.11000]$} \\
\hline LGR & {$[0.0000,0.04000]$} & {$[0.02000,0.06000]$} & {$[0.40000,0.8000]$} \\
\hline GRAC & {$[0.16000,0.12000]$} & {$[0.14000,0.10000]$} & {$[0.12000,0.08000]$} \\
\hline GRCOM & {$[0.14000,0.10000]$} & {$[0.12000,0.08000]$} & {$[0.10000,0.06000]$} \\
\hline
\end{tabular}




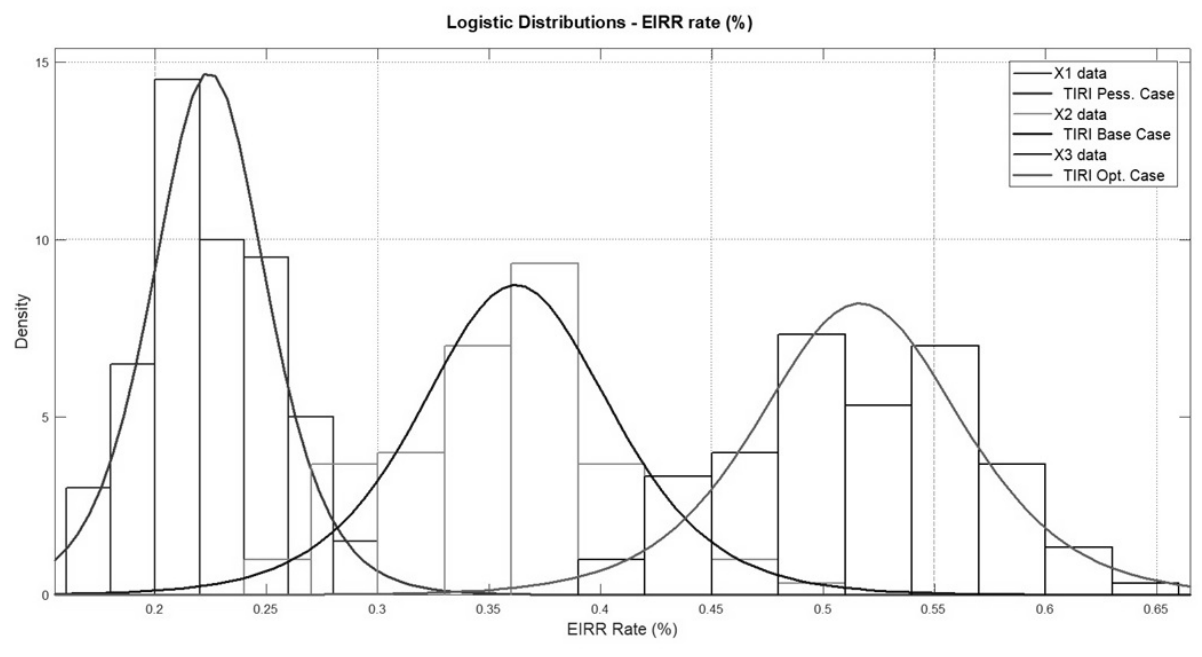

Figure 8: Simulation of EIRR fuzzy financial scenarios

distribution that best represents the EIRR in accordance with the structure of the $S_{-} F L M M$ is the Logistic distribution. In this way, the mean of the EIRR reaches similar values to those obtained by the model after the evolution process as shown in Table 12 and Figure 8. In Table 12, it can be observed that the mean experimented a variation close to $0.57847 \%$, what is a low variation taking into account the magnitude of the EIRR. This fact allows investors to achieve higher credibility values in financing this type of projects, when decisions are made on multi-rates with different effects on the EIRR. In general, the results show a series of characteristics that make $S \_F L M$ the ideal model for the representation of financial scenarios, integrating in a single model following elements: The structure of the linguistic random variables, the causality relationships defined in terms of the structure and form of the sets for each of the input variables (credibility matrix), and also in terms of its iterative mechanism that allows to reach equilibrium points when evaluating the success of a financial scenario. This makes a big difference with respect to traditional models for the construction of financial scenarios, since the model allows the qualitative description of scenarios in a natural way.

Table 11: EIRR probability distribution

\begin{tabular}{|c|c|c|c|c|c|c|c|c|c|}
\hline & LogL & KS(value) & $\mathrm{H}(\mathrm{KS})$ & LogL & KS(value) & $\mathrm{H}(\mathrm{KS})$ & LogL & KS (value) & $\mathrm{H}(\mathrm{KS})$ \\
\hline \hline Normal & 212.05 & 0.10 & not rejected & 158.59 & 0.06 & not rejected & 153.55 & 0.06 & not rejected \\
\hline Cauchy & 191.18 & 0.11 & not rejected & 140.18 & 0.08 & not rejected & 131.71 & 0.10 & not rejected \\
\hline Logistic & 209.72 & 0.09 & not rejected & 156.76 & 0.05 & not rejected & 151.25 & 0.06 & not rejected \\
\hline Beta & 212.87 & 0.09 & not rejected & 158.53 & 0.07 & not rejected & 153.72 & 0.06 & not rejected \\
\hline Exponential & 48.97 & 0.52 & rejected & 1.76 & 0.51 & rejected & -33.77 & 0.54 & rejected \\
\hline Chi-square & -27.77 & 0.55 & rejected & -57.97 & 0.53 & rejected & -80.03 & 0.53 & rejected \\
\hline
\end{tabular}

Table 12: EIRR probability distribution and scenarios

\begin{tabular}{|c|c|c|c|}
\hline & Pessimistic & Base & Optimistic \\
\hline \hline Distribution & Logistic & Logistic & Logistic \\
\hline Log-likelihood & 209.72 & 140.18 & 131.71 \\
\hline Mean & $22.0156 \%$ & $36.746 \%$ & $50.353 \%$ \\
\hline Variance & 0.099 & 0.239 & 0.319 \\
\hline$\%$ Variation & 0.45149 & 0.65041 & 0.63352 \\
\hline Domain & $(-\infty, \infty)$ & $(-\infty, \infty)$ & $(-\infty, \infty)$ \\
\hline
\end{tabular}




\section{Conclusions and future work}

In this paper, we presented a Stochastic Fuzzy Logistic Map $\left(S \_F L M\right)$, inspired by the FCM structure for the modelling of financial scenarios. This model can be used as a support for investment decision making when financing infrastructure systems, where different critical variables intervene. The S_FLM model consolidates in one single structure the characterization of the variables as linguistic rates, and the adaptive multilinear causalities between linguistic input and output variables. It uses an adaptive multilinear model and a fuzzy credibility matrix for establishing a series of partial relations among the fuzzy sets that compose the linguistic variables. This fact allows the modelling of financial scenarios in a holistic way.

The proposed $S_{-} F L M$ model has been created to overcome the limitations imposed by traditional financial models when modelling of dynamic financial scenarios, as, by its conception, the $S \_F L M$ model integrates an evolutionary process that allows for getting a better understanding of behaviour of the EIRR over time. This way achieving IOA values close to $80 \%$ on average among the variables that define a scenario before and after a financing process. The convergence of EIRR towards a much higher rate was mainly due to the fuzzy credibility matrix that allows stabilizing the estimation of EIRR over time for a given financing period. This makes the model an ideal tool for the modelling of dynamic financial scenarios.

The experimental design that was applied for this study allowed the evaluation of the stability of the proposed model with regard to the representation of multi-variable financial scenarios. Here, the structural stability has been substantiated through the structure and the form of the fuzzy sets in the representation of the input variables as linguistic random variables, according to their effect on the EIRR. The dimensional stability has been evidenced through the equilibrium point achieved by the EIRR for each scenario, which took values that were in accordance with the rates that define each scenario. This shows that the general structure of the model is in harmony with the principles that govern the design of scenarios that define the financing of infrastructure systems integrating multiple rates.

Hence, this paper contributes to the body of knowledge and practice because it introduces a new methodology for sampling linguistic variables by using the Monte Carlo method for modelling structure and shape of fuzzy sets that understand the variables as linguistic rates. This sampling process allowed to characterize the input variables as linguistic random rates, establishing a new concept in the representation of probability distributions as linguistic variables, as outlined in Definitions 4 and 5 . Based on these definitions, the uncompressed fuzzy sets with extended slopes showed a major variation with respect to the EIRR, unlike the compressed linguistic rate, which showed a lower variation with regard to the EIRR.

The proposed model also constitutes as an integral tool for teaching project financing through the development of multiple dynamic scenarios. This is owed to the ability of fuzzy neural models to establish complex relations between input and output variables and the flexibility presented by the input variables LAFs for the qualitative description (linguistic variables) and their impact on the EIRR.

To broaden the scope of the present study, our recommendation is to research on topics related to implementing an automatic selection system of the best scenarios for financing an infrastructure system. Such as the integration of the expertise of decision-making specialists within the $S \_F L M$ using Electre-type or AHP models. Additionally, the proposed model can also be extended for modelling dynamic financial scenarios in different knowledge areas because the learning and adaptation capability that allows for automatically modelling input and output variables as linguistic variables to describe the evolution that defines a specific financial scenario. This is why we see, significant potential in both, academic and practical areas for continuing in the development of this body of knowledge [44, 55].

\section{Acknowledgements}

The authors would like to thanks the anonymous referees for providing constructive comments and suggestions. 


\section{References}

[1] Glykas, M., 2012. Performance measurement scenarios with fuzzy cognitive strategic maps. International Journal of Information Management 32 (2), 182-195.

[2] Chang, C.-L., Jimenez-Martin, J.-A., Maasoumi, E., McAleer, M., Prez-Amaral, T., 2019. Choosing expected shortfall over var in basel iii using stochastic dominance. International Review of Economics \& Finance 60, 95 - 113.

[3] Laufer, A., Shapira, A., Telecom, D., 2008. Communicating in dynamic conditions: How do onsite construction project managers do it? Journal of Management in Engineering 24 (2), 75-86.

[4] Findley, T. S., 2014. Using ms excel to solve and simulate the life-cycle/permanent-income model of consumption and saving. International Review of Economics Education 16, 129-146.

[5] Lavipour, S. M. R., Arditi, D., 02 2019. Time-cost tradeoff analysis with minimized project financing cost. Automation in Construction 98, 110-121.

[6] Medda, F., Carbonaro, G., Davis, S., 2013. Public private partnerships in transportation: Some insights from the european experience. IATSS Research 36 (2), 83-87.

[7] Gonzalez-Ruiz, J., Echeverri, S., Ospina, D., 06 2014. Propuesta de un modelo financiero para la evaluación de la inversión en un aeropuerto bajo asociación público-privada. Revista Lmpsakos $11,29-41$.

[8] Tei, A., Ferrari, C., 2018. PPIs and transport infrastructure: Evidence from Latin America and the Caribbean. Journal of Transport Geography 71 (C), 204-212.

[9] Borgonovo, E., Gatti, S., Peccati, L., 2010. What drives value creation in investment projects? an application of sensitivity analysis to project finance transactions. European Journal of Operational Research 205 (1), 227-236.

[10] Gopalan, R., Mukherjee, A., Singh, M., 2016. Do debt contract enforcement costs affect financing and asset structure? The Review of Financial Studies 29 (10), 2774-2813.

[11] Yori Conill, L., Hernandez de Velazco, J., Chumaceiro Hernandez, A., 2011. Planificacion de escenarios: una herramienta estrategica para el analisis del entorno. Revista Venezolana de Gerencia $16,274-290$.

[12] Gatti, S., 2018. Project Finance in Theory and Practice: Designing, Structuring, and Financing Private and Public Projects. In Project Finance in Theory and Practice. London: Elsevier, Academic Press. Elsevier Science.

[13] Azar, A., Dolatabad, K. M., 2019. A method for modelling operational risk with fuzzy cognitive maps and bayesian belief networks. Expert Systems with Applications 115, 607 - 617.

[14] Benjamin, G., Napoles, G., Falcon, R., Froelich, W., Vanhoof, K., Bello, R., 08 2017. A review on methods and software of fuzzy cognitive maps. Artificial Intelligence Review.

[15] Pena, A., Bonet, I., Lochmuller, C., Chiclana, F., Góngora, M., 2018. Flexible inverse adaptive fuzzy inference model to identify the evolution of operational value at risk for improving operational risk management. Applied Soft Computing 65, 614-631.

[16] Ross, T. J., 2012. Fuzzy logic with engineering applications, 3rd Edition. Wiley and Sons, New Delhi.

[17] Truman, R., 2018. Creating a financial model: A look into your company's soul.

[18] Carnicer, J., Penuelas, J., 2012. The world at a crossroads: Financial scenarios for sustainability. Energy Policy 48, 611-617, special Section: Frontiers of Sustainability. 
[19] Geyer, A., Hanke, M., Weissensteiner, A., 2014. No-arbitrage bounds for financial scenarios. European Journal of Operational Research 236 (2), 657-663.

[20] Gao, G., Mishra, B., Ramazzotti, D., 2017. Efficient simulation of financial stress testing scenarios with suppes-bayes causal networks. Procedia Computer Science 108, 272-284, international Conference on Computational Science, ICCS 2017, 12-14 June 2017, Zurich, Switzerland.

[21] Cuomo, S., Somma, V. D., Sica, F., 2018. An application of the one-factor hullwhite model in an iot financial scenario. Sustainable Cities and Society 38, 18-20.

[22] Benczur, P., Cannas, G., Cariboni, J., Girolamo, F. D., Maccaferri, S., Giudici, M. P., 2017. Evaluating the effectiveness of the new eu bank regulatory framework: A farewell to bail-out? Journal of Financial Stability 33, $207-223$.

[23] Chen, C.-W., Ma, W.-J., 2018. Toward a scenario with complementary stochastic and deterministic information in financial fluctuations. Chinese Journal of Physics 56 (3), 853-862.

[24] Bazilian, M., Nussbaumer, P., Rogner, H.-H., Brew-Hammond, A., Foster, V., Pachauri, S., Williams, E., Howells, M., Niyongabo, P., Musaba, L., o Gallachoir, B., Radka, M., Kammen, D. M., 2012. Energy access scenarios to 2030 for the power sector in sub-saharan africa. Utilities Policy 20 (1), 1-16.

[25] Feuerriegel, S., Neumann, D., 2016. Integration scenarios of demand response into electricity markets: Load shifting, financial savings and policy implications. Energy Policy 96, 231-240.

[26] Silva, F., Teixeira, B., Pinto, T., Santos, G., Vale, Z., 2016. Generation of realistic scenarios for multi-agent simulation of electricity markets. Energy 116, 128-139.

[27] Kishita, Y., McLellan, B. C., Giurco, D., Aoki, K., Yoshizawa, G., Handoh, I. C., 2017. Designing backcasting scenarios for resilient energy futures. Technological Forecasting and Social Change 124, 114-125.

[28] Blum, P., Dacorogna, M., 2006. DFA-Dynamic Financial Analysis. American Cancer Society.

[29] Domenica, N. D., Mitra, G., Valente, P., Birbilis, G., 2007. Stochastic programming and scenario generation within a simulation framework: An information systems perspective. Decision Support Systems 42 (4), 2197-2218, decision Support Systems in Emerging Economies.

[30] Eling, Martin, T. D., 2009. Modeling and management of nonlinear dependencies copulas in dynamic financial analysis. Journal of Risk and Insurance 76 (3), 651-681.

[31] Hirtle, B., Kovner, A., Vickery, J., Bhanot, M., 2016. Assessing financial stability: The capital and loss assessment under stress scenarios (class) model. Journal of Banking \& Finance 69, S35-S55, bank Capital.

[32] Ascione, F., Bianco, N., Stasio, C. D., Mauro, G. M., Vanoli, G. P., 2017. Artificial neural networks to predict energy performance and retrofit scenarios for any member of a building category: A novel approach. Energy 118, 999-1017.

[33] Chen, C., 2012. A self organized neuro fuzzy system for air cargo and airline passenger dynamics modeling and forecasting. IJFSA 2 (2), 36-49.

[34] S. Onsel Sahin, F. Ulengin, B. U., 2004. Using neural networks and cognitive mapping in scenario analysis: The case of turkey's inflation dynamics. European Journal of Operational Research 158 (1), 124-145.

[35] Jiang, X., Mahadevan, S., Yuan, Y., 2017. Fuzzy stochastic neural network model for structural system identification. Mechanical Systems and Signal Processing 82, 394-411. 
[36] Salmeron, J. L., Vidal, R., Mena, A., 2012. Ranking fuzzy cognitive map based scenarios with topsis. Expert Systems with Applications 39 (3), 2443-2450.

[37] Kim, J., Han, M., Lee, Y., Park, Y., 2016. Futuristic data-driven scenario building: Incorporating text mining and fuzzy association rule mining into fuzzy cognitive map. Expert Systems with Applications 57, 311-323.

[38] Arvan, M., Omidvar, A., Ghodsi, R., 2016. Intellectual capital evaluation using fuzzy cognitive maps: A scenario-based development planning. Expert Systems with Applications 55, 21-36.

[39] Kireev, V. S., Smirnov, I. S., Tyunyakov, V. S., 2018. Automatic fuzzy cognitive map building online system. Procedia Computer Science 123, 228-233, 8th Annual International Conference on Biologically Inspired Cognitive Architectures, BICA 2017 (Eighth Annual Meeting of the BICA Society), held August 1-6, 2017 in Moscow, Russia.

[40] Jetter, A. J., Kok, K., 2014. Fuzzy cognitive maps for futures studiesa methodological assessment of concepts and methods. Futures $61,45-57$.

[41] Vergini, E. S., Groumpos, P. P., 2016. A new conception on the fuzzy cognitive maps method. IFAC-PapersOnLine 49 (29), 300 - 304, 17th IFAC Conference on International Stability, Technology and Culture TECIS 2016.

[42] Amirkhani, A., Papageorgiou, E. I., Mohseni, A., Mosavi, M. R., 2017. A review of fuzzy cognitive maps in medicine: Taxonomy, methods, and applications. Computer Methods and Programs in Biomedicine 142, 129 - 145.

[43] Mls, K., Cimlerc, R., JanVascakb, Puheimb, M., 2017. Interactive evolutionary optimization of fuzzy cognitive maps. Neurocomputing 232, 58 - 68, advances in Fuzzy Cognitive Maps Theory.

[44] Alessandri, T. M., Ford, D. N., Lander, D. M., Leggio, K. B., Taylor, M., 2004. Managing risk and uncertainty in complex capital projects. The Quarterly Review of Economics and Finance 44 (5), 751-767, managing Uncertainty and Risk.

[45] Christodoulakis, G. A., 2007. Common volatility and correlation clustering in asset returns. European Journal of Operational Research 182 (3), 1263-1284.

[46] Zhang, Z., Huang, J., 2006. Extremal financial risk models and portfolio evaluation. Computational Statistics \& Data Analysis 51 (4), 2313-2338, nonlinear Modelling and Financial Econometrics.

[47] Donkor, E. A., Duffey, M., 2013. Optimal capital structure and financial risk of project finance investments: A simulation optimization model with chance constraints. The Engineering Economist 58 (1), 19-34.

[48] Gonzalez-Ruiz, J., Duque, E., Pena, A., Jimenez, J., Patino, A., 2018. Methodological proposal of financial modelling using dynamic scenarios from multivariable data tables. Int. J. of Technology Enhanced Learning 10 (3), 161-183.

[49] Pena, A., Bonet, I., Lochmuller, C., Chiclana, F., Góngora, M., 2018. An integrated inverse adaptive neural fuzzy system with monte-carlo sampling method for operational risk management. Expert Systems with Applications 98, 11-26.

[50] Vinuela, P., Leon, I., 2004. Redes de neuronas artificiales: un enfoque practico. Pearson Educacion.

[51] Yingxu, W., Lotfi, Z., Bernard, W., Newton, H., Francoise, B., George, B., Frank, H., Guiming, L., Fumio, M., Shushma, P., Victor, R., Shusaku, T., Wei, W., Du, Z., 2017. Abstract intelligence: Embodying and enabling cognitive systems by mathematical engineering. International Journal of Cognitive Informatics and Natural Intelligence (IJCINI) 11 (1), 1-15. 
[52] Pena, A., Bonet, I., Lochmuller, C., Patino, H. A., Chiclana, F., Góngora, M., 2018. A fuzzy credibility model to estimate the operational value at risk using internal and external data of risk events. Knowledge-Based Systems.

[53] Jayashree, L. S., Palakkal, N., Papageorgiou, E. I., Papageorgiou, K., Nov. 2015. Application of fuzzy cognitive maps in precision agriculture: A case study on coconut yield management of southern india's malabar region. Neural Comput. Appl. 26 (8), 1963-1978.

[54] Lisandro, C., January 2013. Dynamic fuzzy cognitive maps. Mathworks.

[55] Park, O.-H., Seok, M.-G., 2007. Selection of an appropriate model to predict plume dispersion in coastal areas. Atmospheric Environment 41 (29), 6095-6101.

[56] Shukla, S., Mohanty, B. K., Kumar, A., 2018. Strategizing sustainability in ecommerce channels for additive manufacturing using value focused thinking and fuzzy cognitive maps. Industrial Management and Data Systems 118 (2), 390-411. 